\title{
A Slowly Growing Orange Patch on the Cheek: Diagnosis of Lupus Vulgaris 20 Years After Onset of First Skin Changes
}

\author{
Claudia Pföhler (D) - Michael Klotz · Thomas Wehler · Thomas Vogt • \\ Cornelia S. L. Müller
}

Received: July 21, 2016 / Published online: November 25, 2016

(c) The Author(s) 2016. This article is published with open access at Springerlink.com

\begin{abstract}
Tuberculosis is a worldwide occurring disease that affects approximately $20-40 \%$ of the world's population and in particular in developing countries. However, in times of migration, industrialised countries are again being more and more affected. Cutaneous tuberculosis is rare and lupus vulgaris represents its most common form. Preferentially, young adults in developing or low-income countries are affected from cutaneous tuberculosis, which usually occurs in previously sensitized persons with a high
\end{abstract}

Enhanced content To view enhanced content for this article go to http://www.medengine.com/Redeem/ 7527F060124D1F89.

C. Pföhler $(\varangle) \cdot$ T. Vogt · C. S. L. Müller

Department of Dermatology, Saarland University

Medical School, Homburg/Saar, Germany

e-mail: claudia.pfoehler@uks.eu

\section{Klotz}

Institute of Medical Microbiology and Hygiene,

Saarland University Medical School, Homburg/Saar,

Germany

T. Wehler

Department of Internal Medicine V, Saarland

University Medical School, Homburg/Saar,

Germany degree of tuberculin sensitivity and a good immunoresponse. In Europe, more elderly people are involved. We present a case of lupus vulgaris that was diagnosed with a delay of about 20 years after onset of first skin changes.

Keywords: Mycobacterium bovis; Lupus vulgaris; Tuberculosis

\section{INTRODUCTION}

Lupus vulgaris is the most common form of skin tuberculosis resulting from an infection with Mycobacterium tuberculosis or M. bovis [1]. Skin lesions usually affect the face, extremities or mucous membranes (tongue and lips) and result from hematogenous, lymphatic or per continuitatem spread of organ tuberculosis [2]. In rare cases, exogenous inoculation can be causative. In Europe, lupus vulgaris is characteristically a disease of elder people, whereas in developing countries younger persons are mostly concerned. Cases of lupus vulgaris due to $M$. bovis bacillus Calmette-Guérin (BCG) at the sites of previous 
BCG vaccination have been reported [2, 3]. Infections with $M$. bovis ssp. bovis or M. bovis ssp. caprae usually result from direct contact with infected animals or consumption of nonpasteurised milk products from dairy cattle [4].

\section{CASE REPORT}

A 56-year-old female patient, who had been living in Saarland, south-west Germany, since birth, presented first in 2012 with an almost 20-year history of an orange, painless, slowly growing skin infiltrate on her right cheek (Fig. 1a). She was referred by her general practitioner who had documented the first skin lesions 19 years ago which he had rated as chronic eczema. In 2012, a skin biopsy was recommend but not performed as the patient refused further diagnostic procedures and therapy. She presented again in 2015 and reported that the skin lesion on the cheek had been growing centrifugally (Fig. 1b). The lesion measured more than $10 \mathrm{~cm}$ in diameter, showed central atrophy and elevated and scaling borders. Diascopy demonstrated a lupoid, apple jelly-like infiltrate. The earlobe was also affected and mutilated. Skin biopsies from the center and from the border of the lesion showed an unaffected epidermis with some parakeratosis.

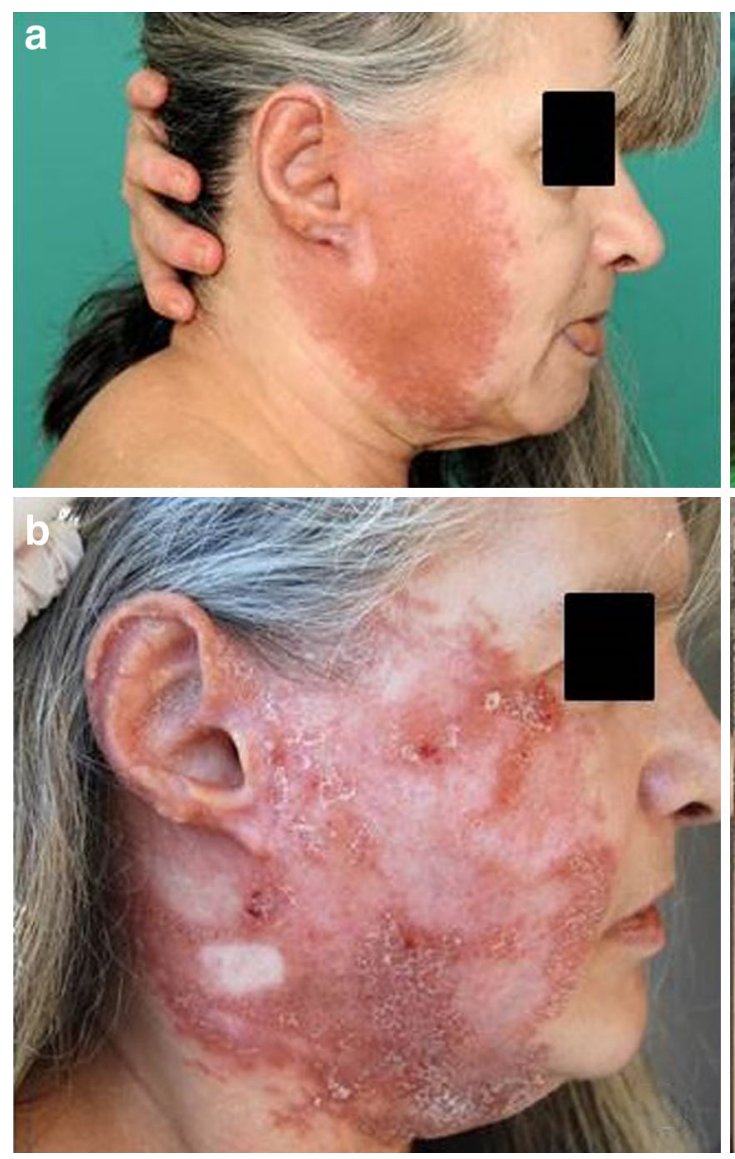

Fig. 1 Skin changes in 2012 when the patient refused further diagnostic procedures (a). Skin lesions in early 2015 prior to therapy (b). Improvement of lupus vulgaris
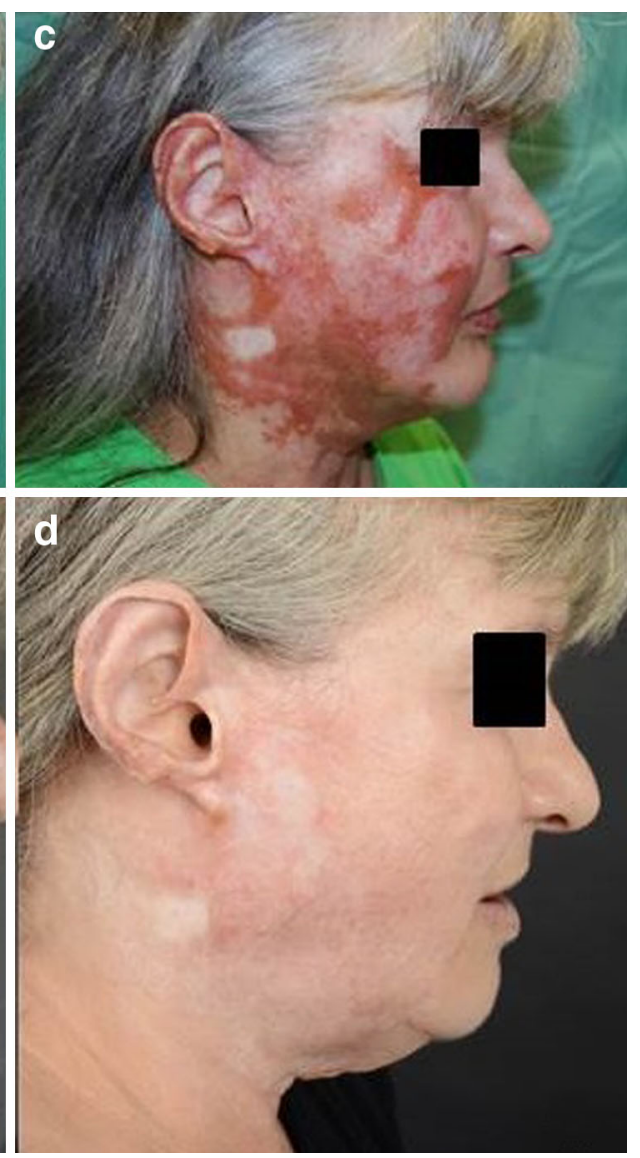

after 6 weeks of antibiotic treatment (c). Complete healing after 1 year of therapy $(\mathbf{d})$ 


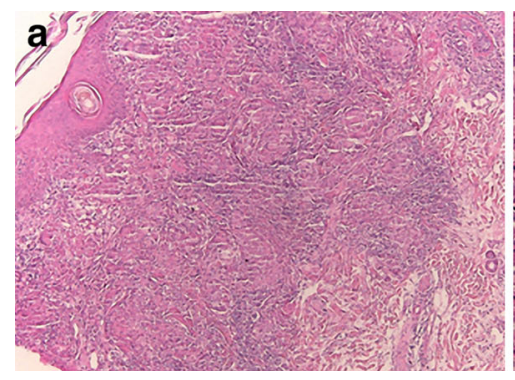

Fig. 2 Histological investigation of a skin biopsy. Dense subepidermal infiltrate of lymphocytic and histiocytic cells with tuberculoid granulomas and central smaller area of caseating necrosis. H\&E staining, $\times 10$ (a). Langerhans

In the dermis, multiple granulomas were present besides epithelioid histiocytes and giant cells with multiple nuclei (Fig. 2a, b). Necrosis and fibroblastic activity could be detected in the center of some of the granulomas (Fig. 2a, b). Ziehl-Neelsen stain showed groups of acid-fast bacilli (Fig. 2c). Conventional culture of fresh biopsy material time-delayed proved Mycobacterium bovis spp. bovis (Geno Type MTBDR by PCR analysis) whereas direct microscopy and the polymerase chain reaction of Mycobacterium complex-specific DNA sequences were negative. The standard QuantiFERON test used as an interferon-gamma release assay to detect a latent tuberculosis was initially negative, even though PPD-specific CD4+ $\mathrm{T}$ cells were detectable in a separate assay. A Mendel-Mantoux test as a tuberculin skin test could not be performed as this test is no longer available in Germany. The search for organ tuberculosis was negative with inconspicuous results in chest X-ray, CT scan of the thorax, bronchoscopy and bronchoalveolar lavage. In conclusion, the diagnosis of lupus vulgaris without systemic involvement was established. Treatment was initiated with isoniazid (300 mg per day), rifampicin (600 mg per day), pyrazinamide (1750 mg per day) and ethambutol (1400 mg per day) at the time of histological confirmation of lupus vulgaris. At giant cells with multiple nuclei, H\&E staining, $\times 40$. Ziehl-Neelsen staining with prove of acid-fast bacilli within a tuberculoid granuloma (arrow), $\times 60$

that time, the results from conventional culture of fresh tissue material was still outstanding. Six weeks later, an improvement of skin infiltration and incipient healing could be observed (Fig. 1c). In the case of M. bovis (Genotype MTBDR), an intrinsic resistance to pyrazinamide must be presumed. However, if an adequate response to therapy can be documented, as in the case presented here, an induction therapy consisting of isoniazid, rifampicin and ethambutol is appropriate. After completion of the induction therapy, an extended maintenance regimen should be continued for a total period of 1 year. Treatment was performed that way in our case and led to complete healing of the lupus vulgaris (Fig. 1d). Additional informed consent was obtained from the patient for whom identifying information is included in this article.

\section{DISCUSSION}

Bovine tuberculosis was a common infection in cow in the 1950s. The infection of humans occurs through the airways or food intake, commonly from unpasteurised cow milk $[4,5]$. The patient presented here confirmed drinking unpasteurized cow milk in her childhood. As she denied any direct contact with cows and 
calves, the consumption of milk from a Tbc infected cow is the most likely method of infection. She reported having never suffered from organ tuberculosis, and clinical investigation showed no signs of organ involvement. She could not remember being BCG-vaccinated. There was no history of tuberculosis in her family nor contact with high-risk persons. It is remarkable that she presented skin lesions in her face for approximately 20 years without being diagnosed with skin tuberculosis, although she was seen regularly by her general practitioner due to depression, a poorly controlled diabetes mellitus type II and hypertension. She reported that the slowly growing skin lesions on her cheek had been interpreted as "a kind of eczema". This was confirmed by her general practitioner. The skin lesions she presented had the typical clinical signs of lupus vulgaris with an orange aspect, and were found in a typical anatomical site involving the cheek, ear and neck. Skin biopsy was able to confirm our tentative diagnosis and culture of fresh biopsy material proved M. bovis spp. bovis, although culture of fresh tissue is often negative, as the PCR diagnostic from paraffin-embedded specimen often is and indeed was in the case presented here [6]. The QuantiFERON test as an interferon-gamma release assay was also negative, which is a rather common phenomenon in diabetic patients, who show a reduced sensitivity to this test [7]. The case presented here should remind treating physicians that tuberculosis still remains a problem in industrialised countries.

\section{CONCLUSION}

Skin tuberculosis is often diagnosed years after the onset of first skin changes. Even in developed countries such as Germany, treating physicians should bear in mind that lupus vulgaris has not disappeared and may still occur, especially in older European patients. Possibly, in times of migration, tuberculosis as well as skin tuberculosis will cause a significant increase in morbidity in high-income countries which accommodate large numbers of refugees and asylum-seekers who have fled from countries in which tuberculosis is still endemic, such as Afghanistan, Pakistan or North African countries like Tunisia [8-11]. In these cases, predominantly young people are affected.

\section{ACKNOWLEDGEMENTS}

No funding or sponsoring was received for this study or publication of this article. All named authors meet the International Committee of Medical Journal Editors (ICMJE) criteria for authorship for this manuscript, take responsibility for the integrity of the work as a whole, and have given final approval of the version to be published.

Disclosures. Claudia Pföhler, Michael Klotz, Thomas Wehler, Thomas Vogt and Cornelia S. L. Müller have nothing to disclose.

\section{Compliance with Ethics}

Guidelines. Additional informed consent was obtained from the patient for which identifying information is included in this article.

Open Access. This article is distributed under the terms of the Creative Commons Attribution-NonCommercial 4.0 International License (http://creativecommons.org/licenses/ by-nc/4.0/), which permits any noncommercial use, distribution, and reproduction in any medium, provided you give appropriate credit 
to the original author(s) and the source, provide a link to the Creative Commons license, and indicate if changes were made.

\section{REFERENCES}

1. Bravo FG, Gotuzzo E. Cutaneous tuberculosis. Clin Dermatol. 2007;25(2):173-80.

2. van Zyl L, du Plessis J, Viljoen J. Cutaneous tuberculosis overview and current treatment regimens. Tuberculosis (Edinb). 2015;95(6):629-38.

3. Walker SL, Lozewicz S, Sood R, Mann TA, Campalani E, Hubbard VG. Lupus vulgaris due to Mycobacterium bovis bacillus Calmette-Guerin (BCG) at the site of previous BCG vaccination. Clin Exp Dermatol. 2009;34(7):e213-5.

4. Tar SY, Bozdemir B, Gurel MS, Bilgin FU, Baran MF, Demirkesen C. Mycobacterium bovis caprae: a rare agent of lupus vulgaris. Clin Exp Dermatol. 2009;34(4):532-3.

5. Meyer S, Naumann L, Landthaler M, Vogt T. Lupus vulgaris caused by Mycobacterium bovis ssp. caprae. Br J Dermatol. 2005;153(1):220-2.
6. Marchetti G, Gori A, Catozzi L, Vago L, Nebuloni M, Rossi MC, et al. Evaluation of PCR in detection of Mycobacterium tuberculosis from formalin-fixed, paraffin-embedded tissues: comparison of four amplification assays. J Clin Microbiol. 1998;36(6):1512-7.

7. Choi JC, Jarlsberg LG, Grinsdale JA, Osmond DH, Higashi J, Hopewell PC, et al. Reduced sensitivity of the QuantiFERON $((\mathrm{R}))$ test in diabetic patients with smear-negative tuberculosis. Int J Tuberc Lung Dis. 2015;19(5):582-8.

8. Abdelmalek R, Mebazaa A, Berriche A, Kilani B, Ben Osman A, Mokni M, et al. Cutaneous tuberculosis in Tunisia. Med Mal Infect. 2013;43(9):374-8.

9. Bua A, Cubeddu M, Piras D, Delogu R, Zanetti S, Molicotti P. Tuberculosis screening among asylum seekers in Sardinia. J Public Health (Oxf). 2016. doi:10.1093/pubmed/fdv215.

10. Ibrahim KM, Khan S, Laaser U. Tuberculosis control: current status, challenges and barriers ahead in 22 high endemic countries. J Ayub Med Coll Abbottabad. 2002;14(4):11-5.

11. Pareek M, Greenaway C, Noori T, Munoz J, Zenner D. The impact of migration on tuberculosis epidemiology and control in high-income countries: a review. BMC Med. 2016;14:48. 